\title{
Vulnerability functions and the influence of seismic design parameters on initial costs for buildings provided with hysteretic energy-dissipating devices
}

\author{
J. García-Pérez, M. Zenteno \& O. Díaz \\ Instituto de Ingeniería, Edificio 2, Mecánica Aplicada, UNAM, \\ Ciudad Universitaria, Mexico
}

\begin{abstract}
This paper is intended to attain both the influence of seismic design parameters on initial cost and seismic vulnerability functions for reinforced concrete buildings provided with hysteretic energy-dissipating devices. In order to obtain this, an established methodology for earthquake resistant design is applied to different types of buildings.

In the optimization process, in order to attain optimum design values, it is necessary to have both initial cost functions, as well as costs due to earthquakes. Initial cost functions are described in terms of design parameters, usually the seismic design coefficient or the vibration period. The influence of seismic design parameters on the initial cost is first studied, and then functions relating costs of the structures to the design parameters are obtained. In order to do this, we analyze different types of reinforced concrete buildings where each one is represented by a reinforced concrete frame composed of beams and columns, with hysteretic energy-dissipating devices installed as braces. The structures studied are hypothetical buildings built at a soft site in the Valley of Mexico with a different number of stories. Cost analyses obtained for these systems are compared with those attained for a conventional frame just composed of beams and columns. Vulnerability functions (drift-seismic intensity) are obtained from those structures studied here. These vulnerability functions together with the cost analyses performed are used to find the cost of damage-seismic intensity relations. The results show that the use of systems with energy-dissipating devices gives a better cost-benefit behavior when the system is under high seismic intensities. Moreover, these results are appropriate for performing longterm cost-benefit analyses.
\end{abstract}

Keywords: energy dissipation, costs, vulnerability curves, reinforced concrete buildings. 


\section{Introduction}

The current seismic design of reinforced concrete buildings is based on the development of structural capacity to dissipate energy due to the action of the acting loads, through elastic or inelastic deformations, thereby developing more efficient levels of structural control every day. One way to attain this structural control is by the so called passive control consisting of increasing structural damping, modifying the natural period of vibration or combining both structural properties. One of the devices applied to a structure requiring this type of control is known as energy dissipating device (EDD). Here we use the type of EDD installed as an external brace to the reinforced concrete frame, linked by stiffeners working in tension or compression. These devices are manufactured in a factory, thus maintaining appropriate quality control, and providing stable hysteretic behavior under high cycles of deformations. The foregoing provides high capacity for dissipating energy. Failure conditions of these elements are obtained at laboratories by studying their load deformation capacity.

On the other hand, in order to attain optimum design parameters for buildings erected in seismic zones, the current optimization philosophy requires balancing the total expected present cost of a structure, including the initial cost and maintenance costs, as well as losses due to damage and failure. Regarding initial costs in terms of the base shear design coefficient, we find some expressions in the literature developed by Whitman et al [1], Grandori [2], Ferrito [3], Rosenblueth [4], Vargas and Jara [5], and García-Pérez [6]. Some studies have also been done in obtaining initial costs functions in terms of the natural period of vibration of the structure (Reyes [7], Esteva et al [8], García-Pérez et al [9]). Despite the studies carried out so far, some expressions are still necessary for initial costs in terms of design parameters, especially for structures with energy dissipating devices. Here we find some expressions in terms of the basal shear coefficients, as well as the period of vibration for both conventional structures and structures with EDDs. Then the initial cost expressions are used to obtain functions describing probable damages to the structures in terms of the intensity of the seismic motion causing these damages.

\section{Buildings with energy dissipating devices}

The purpose of seismic design is to provide each structure with characteristics allowing for developing optimum behavior in terms of their design economy, when subjected to the action of earthquakes occurring during their life-cycle. Satisfactory structural behavior is expected under low intensity seismic events, since story displacements are controlled to minimize damage in nonstructural elements. Under strong seismic excitations, structural collapse through damage of some structural elements must be avoided by controlling their deformations.

A balance between economy and structural safety must be pursued, although allowable limits of linear behavior are exceeded in some members, but without reaching failure. Therefore, the structures proposed here are ductile spatial frames able to resist lateral forces through their stiffness and energy dissipating 
devices. Ductile behavior of structures is closely linked to damage of their structural elements, thus research is directed at mixed systems comprised of reinforced concrete and EDDs, trying to concentrate dissipated energy mostly by reducing deterioration and degradation of mechanical and dynamical properties of conventional structural elements.

Seismic design criteria for reinforced concrete ductile frames established in the Federal District Building Code and its Complementary Technical Norms (RCDF) [10], consider that all structural elements under high intensity seismic excitation undergo inelastic deformations, when absorbing and dissipating a fraction of the total energy acting in the whole structure. These deformations are concentrated in specific regions of frames, such as the zones of maximum internal moments. Seismic design criteria are intended to avoid structural collapse by designs based on weak beams and strong columns, ensuring that under highly seismic excitations larger inelastic deformations will occur at the end of beams and not in the columns.

\subsection{Methodology in buildings with EDDs}

In order to design the buildings with EDDs studied here a methodology developed by Campos [11] is used. This methodology is based on a performance design criterion using allowable ductilities and a group of parameters that influence greatly on the behavior of the structural systems. These parameters are related each other during the different steps of the analysis and design.

The energy induced on a structure depends on different factors which are directly related to soil motion, damping, stiffness and strength, among other things. On the other hand, structural response under seismic excitation can be improved by either decreasing input seismic energy or by increasing dissipating energy. The latter may be reached by introducing viscous or hysteretic damping. The methodology developed by Campos [11] consists of obtaining stiffness and strength for each story without exceeding both allowable deformations and ductilities as well as developing the ductility established for the design.

\subsection{Variables used in the design process}

In the design methodology adopted, design variables denominated as control variables are those design parameters defining mechanical properties of the structural system, as well as maintenance and repairing politics. Lateral stiffness of the dissipating device $k_{d}$ related to total stiffness $K$ in each building story is denoted by $r_{k}=k_{d} / K$. Yielding displacement of the dissipating device $\delta_{y d}$ related to yielding displacement of the conventional frame $\delta_{y c}$ is represented by $\varphi=\delta_{y d} / \delta_{y c}$. Relationship between lateral strength of the dissipating device $R_{d}$ to total lateral strength $R$ of each story is given by $r_{R}=R_{d} / R=\varphi r_{k} / 1+(\varphi-1) r_{k}$. If we consider the same maximum lateral displacement of the story for both conventional frames and frames with EDDs, it is found that the relationship between ductilities under lateral displacement of a story for each case is given 
by $\varepsilon=\mu_{d} / \mu_{c}=1 / \varphi$. From the definition of control variables, the following equations were obtained: $\alpha=r_{k} / 1-r_{k}=k_{d} / k_{c}, \beta=\varphi \alpha=R_{d} / R_{c}$, where $k_{c}$ and $R_{c}$ are the stiffness and lateral strength of the story for the conventional frame. Expressions for total stiffness and strength are given by $K=k_{c}+k_{d}=k(1+\alpha)$; $R=R_{c}+R_{d}=R_{c}(1+\beta)$. In order to apply a superposition between the conventional frame and the system with EDDs it is necessary that $\mu=\delta_{\max } / \delta_{y}=\delta_{\max } K / R$, where $\delta_{y}$ and $\delta_{\max }$ are the yielding and maximum relative displacements of the story of the frame with EDDs. A relationship between elastic and inelastic spectral ordinates is expressed as $Q(\mu, T)=S_{e}(T) / S(\mu, T)=c_{e} / c_{i}$, where $T$ stands for the structural period, and $c_{e}$ and $c_{i}$ denote the elastic and inelastic seismic design coefficient.

\section{Seismic design procedure}

\subsection{Design spectrum}

The design spectrum used here is an average spectrum normalized to the spectral intensity of a family of five accelerograms, with statistical properties similar to those of the record obtained for the September 19, 1985 earthquake at SCT site in Mexico City (García-Pérez et al [9]). Normalization of this spectrum consists in equating intensities of the elastic spectra of the earthquakes simulated to those intensities of the earthquake recorded. The spectral intensity of each normalized spectrum accelerations is defined by $(1 / 2 \pi) \int_{0}^{T_{1}} S_{a}(T, \xi) \mathrm{d} T$, where $S_{a}(T, \xi)$ is the spectral acceleration ordinate corresponding to structural period $T$, with damping $\xi=0.05$ and $T_{1}=3.5 \mathrm{sec}$. for earthquakes occurring in soft soil.

\subsection{Procedure}

Here we adopt the design process developed by Campos [11] consisting of a preliminary step or design and a final design. In the first step, values for variables $\alpha$ and $\beta$ are computed taking into account the most appropriate level of ductility, and also maximum story displacements $\delta_{\max }(1.2 \%$ of the height story) are determined. Then an estimation of the structural period is done by taking it as ten per cent of the total number of stories, and the corresponding elastic $\left(c_{e}\right)$ and inelastic $\left(c_{i}\right)$ coefficients are obtained with the aid of the design spectrum. Seismic forces and corresponding design shear force $R_{e}$ are determined by means of a seismic static analysis, and the reduced design shear force $R$ is obtained as $R=R_{e} / Q=R_{e} \gamma \mu$. With these data, values of the control variables are proposed as a percentage of stiffness and strength that the EDD must provide. Then a relationship between ductilities is found such that it will be our limit parameter 
of comparison between the structural design of the conventional frame and the frame with EDDs. It is also considered that under lateral loads, each story has two significant displacements, namely, a relative horizontal displacement $\delta_{H}$, and a rotation as a rigid body $\theta$ resulting from elongation and contraction of columns, thus the story deformation is given by $\delta=\delta_{H}-\theta h$. Structural elements of the frame with EDDs are pre-designed in terms of stiffness and strength required according to the percentage that each system must provide (frame and EDD), thus successive iterations are performed until finding more appropriate dimensions giving displacements similar to those allowed. To complete the first step, a comparison between stiffness of the stories is made by coefficient $C_{k}=k_{a} / k_{c}$, which must stay within the permissible limit given by $\left|C_{k}-1\right| \leq \epsilon$, where $k_{a}$ is the new lateral stiffness of the story.

After determining dimensions of structural elements, beams, columns, and EDDs, we proceed to design them, and finally the relative displacements in each story are checked such that allowable limits indicated in the RCDF [10] are complied.

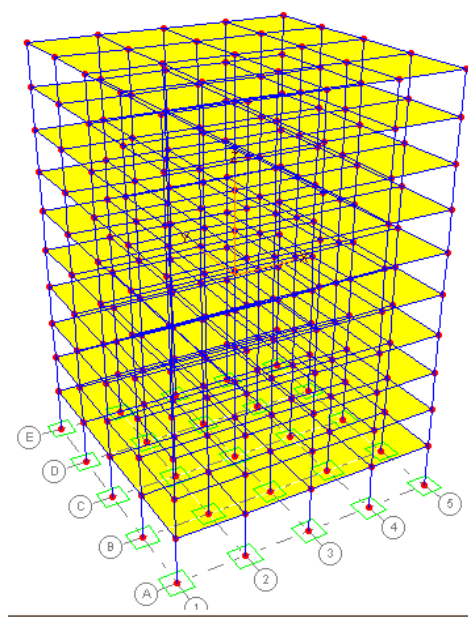

a)

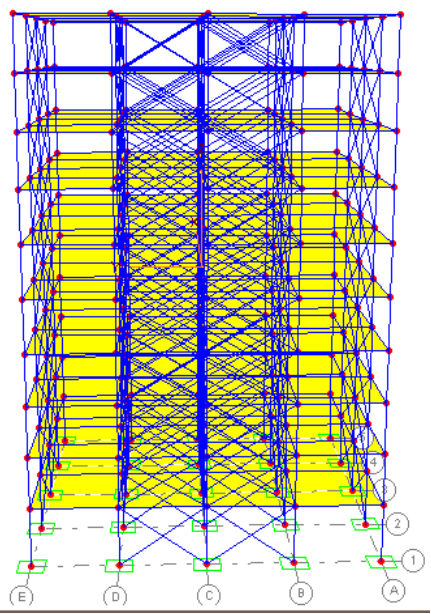

b)

Figure 1: a) Conventional frame; b) frame with EDDs.

\section{Types of structures studied}

A group of regular buildings of five, ten, fourteen, and twenty stories were designed in order to find their initial cost functions. The structures are regular symmetrical frames in both directions, with four bays five meters wide each. The first story is 3.5 meters high while all upper stories are $3 \mathrm{~m}$. high. Compression strength of the concrete $f_{c}^{\prime}=250 \mathrm{~kg} / \mathrm{cm}^{2}$ and yield strength of the 
steel $f_{y}=4200 \mathrm{~kg} / \mathrm{cm}^{2}$ are used. Floor systems are based on reinforced concrete slabs supported by beams and behaving as a rigid diaphragm. Columns are designed for uniaxial bending, as long as we are using bi-dimensional frames. A model of the buildings used is shown in fig. 1 for conventional frames and frames with EDDs, respectively.

Structural analysis of the buildings is performed under a model for plane frames comprised of rigid nodes connecting to flexible bars of finite stiffness. Their behavior resembles that of a real building by taking into account deformations by axial load in columns, as well as deformations due to bending and shear in beams and columns. Loads and load combinations applied to each structural system are determined according to the RCDF [10], resulting a dead load of 560 and $420 \mathrm{~kg} / \mathrm{m}^{2}$ for roof and story in this case, respectively, as well as a live load for gravitational loads of 250 and $100 \mathrm{~kg} / \mathrm{m}^{2}$ for roof and story, and a live load for earthquakes of 180 and $70 \mathrm{~kg} / \mathrm{m}^{2}$.

\subsection{Design of energy dissipating devices}

The energy dissipating devices employed here are steel diagonals A-36 that stiffen the frame and are located diagonally according to the array previously determined, as shown in fig. 1b. These dissipating devices lead to stiffness $K_{D}$ which together with stiffness of the concrete frame $K_{C}$ provides the required design stiffness of the structural system. Cross sectional areas of the diagonals are computed by $A=k_{D} L /\left(E_{D} D \cos ^{2} \theta\right)$ where $L$ is the length of the diagonal, $E_{D}$ is the material modulus of elasticity, $D$ the number of diagonals in each story, and $\theta$ is the angle between the diagonal and the horizontal line. Once the preliminary cross section is obtained, the design forces of the EDDs can be computed. The diagonal elements work under an axial load to both compression and in tension. Designing the connection assumes that the design force induced by seismic motion acts in the direction of the diagonal.

\subsection{Values of some variables}

In order to find the stiffness and strengths of frames and EDDs under study, values of 0.5 are proposed for $\alpha$ and $\beta$ which lead to $K_{d}=0.33, K_{C}=0.67, R_{C}=0.67$ and $R_{d}=0.33$. Now the relationship of yield displacements between EDDs and the corresponding story is given by $\beta / \alpha=1$, such that the relationship between lateral strength of the EDD and total strength of the structural system of each story is given by $r_{k}=R_{d} / R=\psi r_{k} /\left[1+(\psi-1) r_{k}\right]=0.33$. The ductility relationship between the two systems, that is, EDD and conventional frame, is given by $\varepsilon=\mu_{d} / \mu_{c}=1 / \psi$ which becomes 1 if it is assumed that $\mu_{d}=\mu_{c}=3$. Superposition of both systems requires that $\mu=\delta_{\max } / \delta_{y}=\delta_{\max } k / R$. 


\section{Results}

\subsection{Initial cost functions}

After designing conventional buildings and buildings with EDDs, a cost analysis is performed to know their total cost. The analysis takes into account only structural costs (beams, columns and EDDs), but not costs of nonstructural elements, finishing or indirect costs.

In a previous paper by García-Pérez et al [9], initial cost functions in terms of seismic design coefficients were presented, and four types of reinforced concrete buildings were analyzed. Each one of the types corresponds to a period of vibration. Here, we analyze buildings with five, ten, fourteen and twenty stories. For each building type, we designed different structures with different periods of vibration, thereby allowing for initial cost curves in terms of the period of vibration for five, ten, fourteen and twenty stories, as shown in fig. 2. General expressions for initial cost functions in terms of seismic design parameters are still underway.

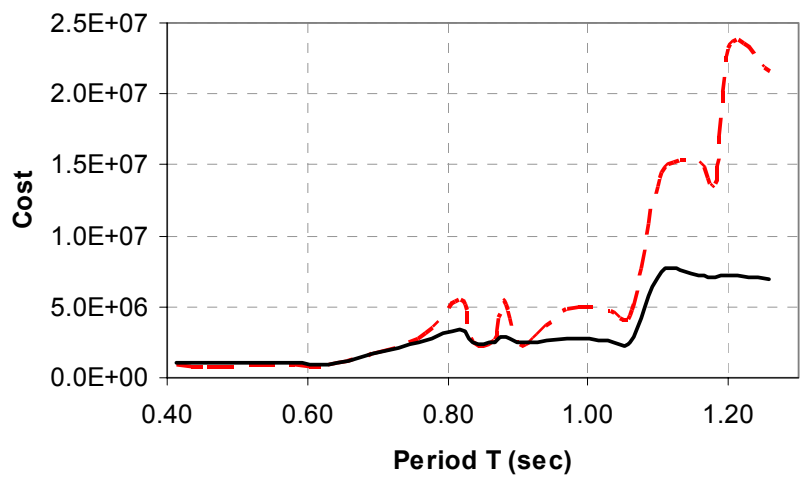

Figure 2: Initial cost in terms of period of vibration for conventional frames (dash line) and frames with EDDs (continuous line).

\subsection{Vulnerability functions}

Seismic vulnerability functions are computed here by following the methodology explained in García-Pérez et al [9], where the vulnerability function in terms of the economic consequences is expressed by the following equation as: $\delta_{E}(y)=\delta_{E}(y \mid S)\left(1-p_{F}(y)\right)+\delta_{E F} p_{F}(y)$, where $\delta_{E}(y)$ is the expected value of the damage cost due to an earthquake of intensity $y, \delta_{E}(y \mid S)$ is the expected value of such cost, but it is conditioned to the survival of the system denoted by $S$, to the intensity $y . \delta_{E F}$ is the cost of collapse and $p_{F}(y)$ is the probability of occurrence of collapse. A detailed explanation of the computation of these functions is given in García-Pérez et al [9]. 


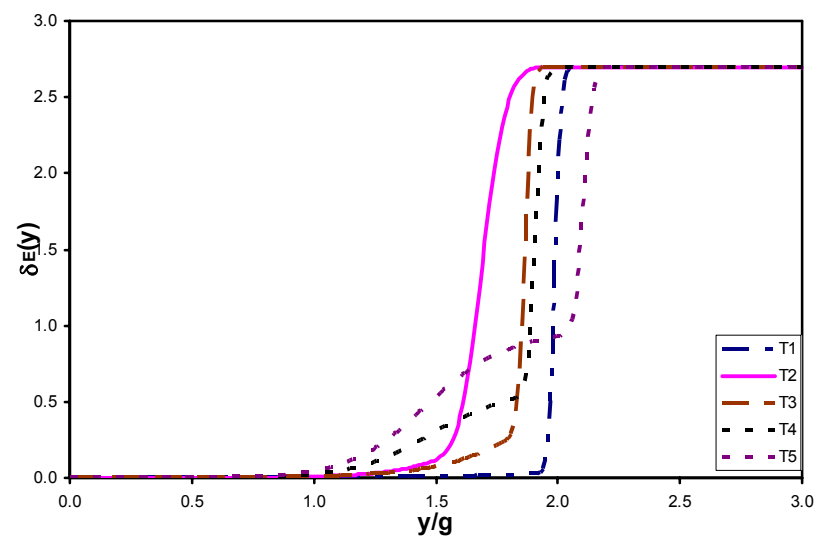

Figure 3: Vulnerability functions for conventional frames with 5 stories.

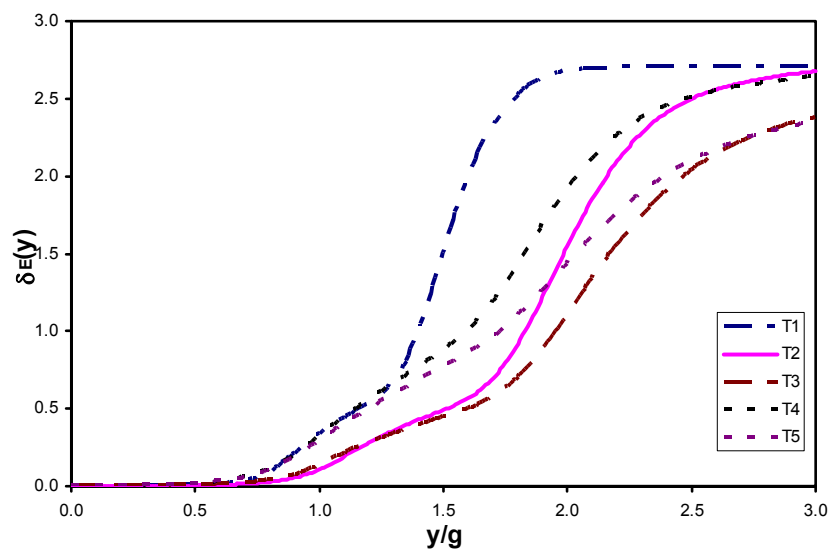

Figure 4: Vulnerability functions for conventional frames with 14 stories.

Figures 3 and 4 show vulnerability functions obtained for conventional buildings of five and fourteen stories, respectively. The results show that for both cases there is no clear trend of the variation of these functions in terms of the period of vibration for the same number of stories. In buildings with five stories the systems with a smaller period of vibration show that the cost of damage increases quickly within a small interval of intensities, reaching the total failure relatively faster once the damage has occurred. As the period of vibration increases, the damage cost with the intensity increases very slowly, although the damage is presented at smaller intensities. On the other hand, in those systems with large periods of vibration, damage in the dividing walls seems to exert certain influence at the beginning. In structures with fourteen stories, the system with a smaller period of vibration, together with the two systems of larger period, 
presents a large increment of damage at lower intensities than those for intermediate systems. Here again, the dividing walls contribute significantly to the damage when it begins. The wide dispersion in vulnerability functions obtained for the systems with the same number of stories, but for different periods of vibration, must be studied in great detail, to determine if the dispersion is due to the fitting of the functions used to compute the vulnerability functions, since we have used very simple approximations in order to solve the problem. Those cases for structures with EDDs are currently under study.

\section{Concluding remarks}

Initial cost curves were obtained in terms of design parameters for both conventional structures and structures with energy dissipating devices. Systems with five, ten, fourteen and twenty stories were analyzed, and different periods of vibration were considered for each one of these structures. The expressions obtained were used in computing the vulnerability functions of the systems which show wide dispersions due perhaps to the use of approximations in the fitting process. General expressions in terms of design parameters and vulnerability functions for systems with energy dissipating devices are still left for future research.

\section{References}

[1] Whitman, R.V., Biggs, J.M., Brennan, J., Cornel C.A., de Neufville, R. \& Vanmarcke, E, Summary of methodology and pilot application. Seismic decision Analysis Report No. 9, MIT Dept of Civil Engineering, Cambridge, MA, Oct, 1973.

[2] Grandori, G., Seismic zoning as a problem of optimization, Proc Second International Conference on Structural Safety and Reliability, Munich, pp 613-624, 1977.

[3] Ferrito, J.M., Economics of seismic design for new buildings, Journal of Structural Engineering, ASCE 110(12), pp 2925-2937, Dec, 1984.

[4] Rosenblueth, E., What should we do with structural reliabilities, Reliability and Risk Analysis of Statistics and Probability in Soil and Structural Engineering, Waterloo, Ontario, pp 24-34, May, 1987.

[5] Vargas, E. \& Jara, J. M., Influencia del coeficiente sísmico de diseño en el costo de edificios con marcos de concreto, Memorias VIII Congreso Nacional de Ingeniería Sísmica, \& VII Congreso Nacional de Ingeniería Estructural, Acapulco, Gro, pp D30-39, Nov, 1989, (In Spanish)

[6] García-Pérez, J., Seismic zoning for initial- and total-cost minimization, Earthquake Engineering \& Structural Dynamics, 29, pp 847-865, 2000.

[7] Reyes, C., The service limit state in the seismic design of buildings, PhD thesis, School of Engineering, National University of Mexico, 1999, (In Spanish) 
[8] Esteva, L., Díaz, O., García-Pérez, J., Sierra, G., \& Ismael, E., Life-cycle optimization in the establishment of performance-acceptance parameters for seismic design, Structural Safety, 24 (2-4), pp. 187-204, 2002.

[9] García-Pérez, J., Zenteno, M., \& Díaz, O., Initial cost and seismic vulnerability functions for buildings with energy-dissipating devices, First International Conference on Safety and Security Engineering, Rome, Italy, WIT Press, 82, pp. 161-170, Jun, 2005.

[10] Federal District Building Code and its Complementary Technical Norms (RCDF), Diario Oficial de la Federación, México, DF, 2004, (in Spanish)

[11] Campos, D., Optimization criteria for the design of buildings with hysteretic energy-dissipating devices, $\mathrm{PhD}$ thesis, National University of Mexico, 2005, (in Spanish) 